\title{
Middle East Respiratory Syndrome Coronavirus (MERS-CoV)
}

\author{
Article by Geethamma Jolly \\ RN-MSN, Texila American University, India \\ E-mail: christy20042001@yahoo.com
}

\begin{abstract}
Middle East respiratory syndrome (MERS) is a highly lethal respiratory disease caused by a novel single-stranded, positive-sense RNA beta coronavirus (MERS-CoV). Dromedary camels, hosts for MERS-CoV, are implicated in direct or indirect transmission to human beings, although the exact mode of transmission is unknown. The virus was first isolated from a patient who died from a severe respiratory illness in June, 2012, in Jeddah, Saudi Arabia. As of May 31, 2015, 1180 laboratoryconfirmed cases (483 deaths; 40\% mortality) have been reported to WHO. MERS-CoV causes severe human infections resulting in high mortality and has demonstrated the ability to transmit between humans. So far, the observed human-to-human transmission has occurred mainly in health care settings. WHO expects that additional cases of MERS-CoV infection will be reported from the Middle East, and that cases will continue to be exported to other countries by individuals who might acquire the infection after exposure to animals or animal products (for example, following contact with dromedaries) or human source (for example, in a health care setting). Both community-acquired and hospital-acquired cases have been reported with little human-to-human transmission reported in the community. Although most cases of MERS have occurred in Saudi Arabia and the United Arab Emirates, cases have been reported in Europe, the USA, and Asia in people who travelled from the Middle East or their contacts. Clinical features of MERS range from asymptomatic or mild disease to acute respiratory distress syndrome and multiorgan failure resulting in death, especially in individuals with underlying comorbidities. No specific drug treatment exists for MERS and infection prevention and control measures are crucial to prevent spread in health-care facilities. MERS-CoV continues to be an endemic, low-level public health threat. However, the virus could mutate to have increased interhuman transmissibility, increasing its pandemic potential.
\end{abstract}

\section{Keywords}

1. Introduction

- Ecology and transmission of MERS-CoV

- Virology

- Origin

- Middle East respiratory syndrome 3-D image

- Tropism

- Transmission

- Evolution

- Natural reservoir

- Taxonomy

2. Transmission Modes

- Possible sources and modes of transmission

- Bats

- Camels

3. Clinical feature

4. Diagnosis

5. Treatment

6. Prevention 
Texila International Journal of Public Health

Volume 4, Issue 4, Dec 2016

7. Imaging findings at presentation in Saudi patients withMERC-CoV

8. Ways of protection against the MERC-CoV

9. Infection Prevention and Control Recommendations for Hospitalized Patients with MERS-CoV

- Recommendations

A. Minimize Chance for Exposures

- Before Arrival

- Upon Arrival and During the Visit

- Ensure Adherence to Standard, Contact and Airborne Precautions

A. Hand Hygiene

B. Personal Protective Equipment

C. Gloves

D. Gowns

E. Respiratory Protection

F. Eye Protection

- Using More than one Kind of Personal Protective Equipment (PPE)

- Patient Placement

- Manage Visitor Access and Movement within the Facility

- Implement Engineering Controls

- Monitor and Manage Ill and Exposed Healthcare Personnel

- Train and Educate Healthcare Personnel

- Implement Environmental Infection Control

- Establish Reporting within Hospitals and to Public Health Authorities

10. MERS-CoVStatistics

11. Comparisons

12. Summary and Recommendations

13. References

\section{Introduction}

Middle East respiratory syndrome (MERS) is a viral respiratory disease caused by a coronavirus (MERS-CoV) that was first identified in Saudi Arabia in 2012. Coronaviruses are a large family of viruses that can cause diseases ranging from the common cold to Severe Acute Respiratory Syndrome (SARS). It is caused by a virus that is different from any other virus that has been previously found in people. Symptoms of MERS include fever, cough, and shortness of breath. CDC is working with the World Health Organization and other partners to understand the public health risks from this virus

The first report of Middle East respiratory syndrome (MERS) described a patient who died from a severe respiratory illness in a hospital in Jeddah, Saudi Arabia, in June, 2012. A previously unrecognized coronavirus (MERS-CoV) isolated from this patient1 was similar to severe acute respiratory syndrome coronavirus (SARS-CoV), which caused an epidemic in 2002-03. The virus was initially designated human coronavirus-EMC, but was renamed MERS-CoV with global consensus. The genomic structure of MERS-CoV was delineated3 and dipeptidyl-peptidase (DPP4, also known as CD26) was identified as the host-cell receptor for cell entry. Reverse genetics enabled the virus's genome to be studied, and molecular diagnostic tests were quickly developed.

The high mortality rates in family-based and hospital-based outbreaks, especially in patients with comorbidities such as diabetes and renal failure, along with the respiratory droplet route of transmission, evoked global concern and intensive discussion in the media. The numbers of reported MERS cases spiked during hospital-based cluster outbreaks in the spring of 2013 and 2014; some cases are still detected throughout the year. MERS-CoV was deemed a serious public health epidemic threat, because millions of pilgrims from 184 countries converge in Saudi Arabia each year for the Hajj and Umrah pilgrimages. Fortunately, no MERS cases were associated with the 2013 and 2014 Hajj pilgrimages. 
The Middle East respiratory syndrome coronavirus (MERS-CoV), is a novel positive-sense, singlestranded RNA virus of the genus Beta coronavirus. Initially called novel coronavirus 2012 or simply novel coronavirus, it was first reported in 2012 after genome sequencing of a virus isolated from sputum samples from person who fell ill in a 2012 outbreak of a new flu.

As of July 2015, MERS-CoV cases have been reported in over 21 countries, including Saudi Arabia, Jordan, Qatar, Egypt, the United Arab Emirates, Kuwait, Turkey, Oman, Algeria, Bangladesh, Indonesia (none were confirmed), Austria, the United Kingdom, South Korea, the United States, Mainland China, Thailand, and the Philippines.

Preventing transmission of respiratory pathogens including MERS-CoV in hospitals requires the application of infection control procedures and protocols including environmental and engineering controls, administrative controls, safer work practices, and personal protective equipment (PPE). Measures that enhance early detection and prompt triage and isolation of patients who should be evaluated for MERS-CoV (Interim Guidance for Healthcare Professionals) are critical to ensuring effective implementation of infection control measures. Successful implementation of many, if not all, of these strategies is dependent on the presence of clear administrative policies and organizational leadership that promote and facilitate adherence to these recommendations among the various people within the healthcare setting, including patients, visitors, and HCP.

Between 19-22 June 2016, WHO published 3 reports on the Disease Outbreak News (DON) describing 25 cases of Middle East Respiratory Syndrome (MERS). Twenty-four of the cases were identified as having contact with a probable, single index case who was diagnosed with MERS in a hospital in Riyadh City, Riyadh Region. The 22 June 2016 DON reported that the index case had died.

Based on available information, the probable index case is a woman who presented on 10 June 2016 to the hospital with a critical health condition, not consistent with MERS symptoms. She was triaged in the emergency room of the hospital and admitted to the vascular surgery ward. Following admission the patient showed signs of respiratory illness, and MERS was suspected. The hospital diagnosed and confirmed MERS on 12 June 2016, within 48 hours of her original admission. The patient's MERS clinical symptoms were initially masked by other predominant symptoms.

Immediately following diagnosis the Ministry of Health of Saudi Arabia dispatched a rapid response team to the hospital. The team conducted active screening and contact tracing to identify health care workers, visitors, patients and household contacts who may have been exposed to MERS by the probable index patient. In addition, other public health control measures were immediately implemented in the hospital to limit further transmission.

As of 22 June 2016 twenty-four (24) contacts have tested positive for MERS including twenty (20) healthcare contacts and three (3) household contacts. In addition, one case has been diagnosed in a household contact of a hospital patient who was diagnosed with the disease after exposure to the probable index case. Twenty (20) of the twenty-four (24) have not exhibited any MERS symptoms.

According to WHO General-Director Margaret Chan "the Kingdom of Saudi Arabia has worked to improve its response to this challenging disease, especially through infection prevention and control in health care facilitates. Diagnosis is often complicated in patients affected by multiple comorbidities."

Globally since September 2012 WHO has been notified of over 1,700 laboratory-confirmed cases of infection with MERS, in 27 countries, including more than 600 related deaths.

Based on the current situation and available information, WHO encourages all Member States to continue their surveillance for acute respiratory infections and to carefully review any unusual patterns.

Infection prevention and control measures are critical to prevent the possible spread of MERS in health care facilities. It is not always possible to identify patients with MERS early because like other respiratory infections, the early symptoms of MERS are non-specific. Therefore, health care workers should always apply standard precautions consistently with all patients, regardless of their diagnosis. 
Droplet precautions should be added to the standard precautions when providing care to patients with symptoms of acute respiratory infection; contact precautions and eye protection should be added when caring for probable or confirmed cases of MERS infection; airborne precautions should be applied when performing aerosol generating procedures.

\section{Ecology and transmission of MERS-CoV}

MERS-CoV might have originally spread from bats to camels and other, as yet unidentified, intermediate hosts. The virus has circulated in camel populations in Africa and the Arabian Peninsula for at least 20 years. In 2012, MERS-CoV spread to human populations, with camels the most likely source. Several possible routes of spread from camels to humans exist. MERS-CoV is believed to be transmitted among human beings by droplet, contact, and perhaps airborne spread. MERS manifests in people in various ways, ranging from asymptomatic to fulminant infections. Patients with underlying disease such as diabetes or kidney or liver disease or who are immunocompromised develop more severe disease and have a higher mortality rate after infection. Image credits: camel birthing from Noboru Komine/Science Photo Library, camel milking from ACEI Cheung/CC BY-SA 2.0, camel owner contact from Peter Menzel/Science Photo Library, MERS virus from AMI Images/Science Photo Library.

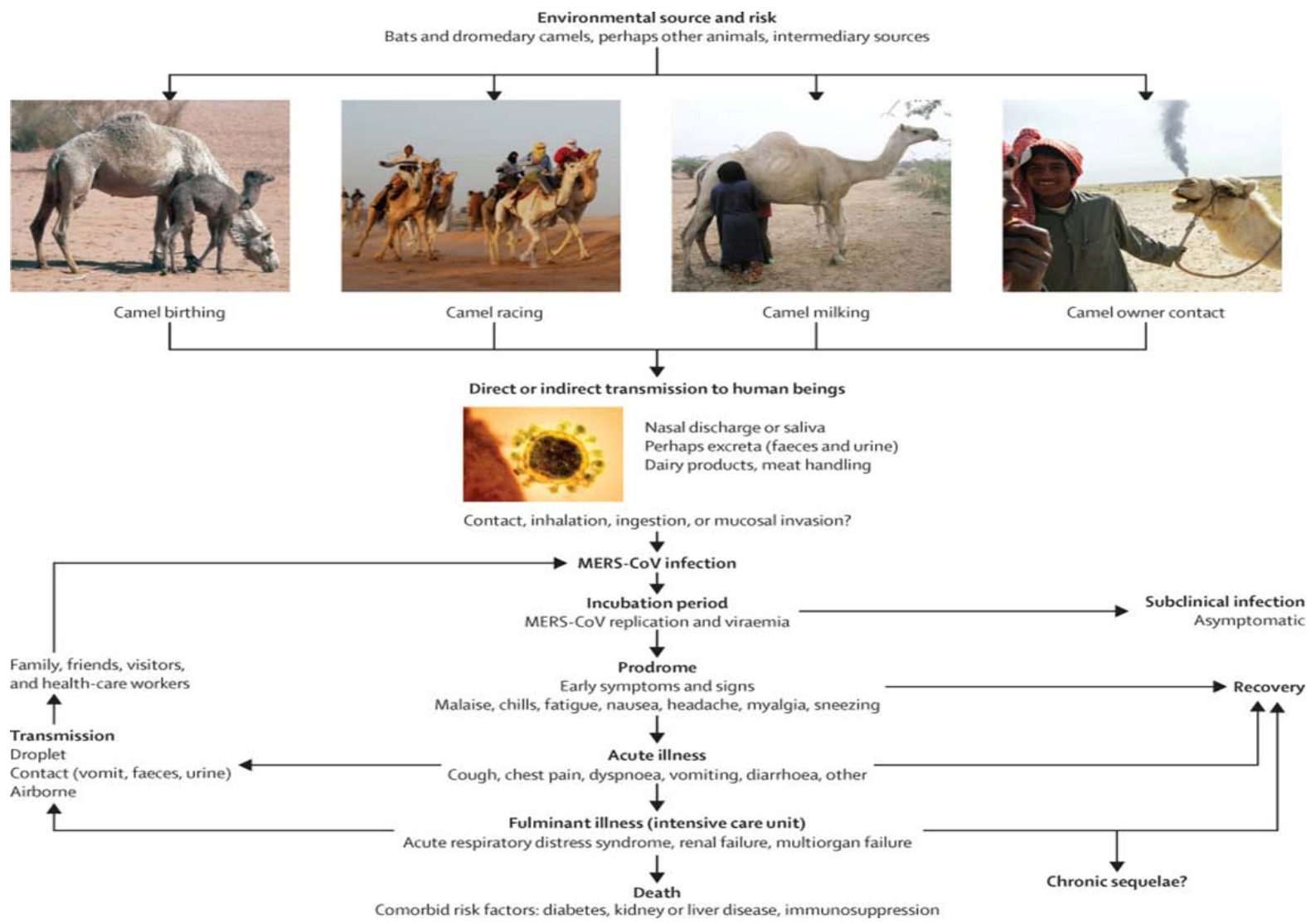

\section{Virology}

Coronaviruses are large (28-32 kb) single-stranded positive-sense RNA viruses. To enter host cells, MERS-CoV attaches to its receptor, dipeptidylpeptidase. Protease cleavage of the $\mathrm{S}$ protein is then required for virus-cell fusion and release of genomic RNA into the cytoplasm. Viral RNA transcription and replication occurs on double membrane vesicles and other membranous structures, which are derived from the endoplasmic reticulum. Transcription of the seven sub genomic mRNAs occurs via negative-strand subgenomic RNA intermediate 
The virus MERS-CoV is a new member of the beta group of coronavirus, Beta, lineage C. MERS$\mathrm{CoV}$ genomes are phylogenetically classified into two clades, clade A and $\mathrm{B}$. The earliest cases of MERS were of clade A clusters and new cases are genetically distinct (clade B).

The genomic structures of the two viruses are very similar, with proteins involved in virus replication encoded at the $5^{\prime}$ end and structural proteins encoded by genes at the 3 ' end of the genome. Accessory proteins, which are not required for virus viability, are interspersed throughout the structural genes and might interfere with the innate immune response in infected animals. MERS-CoV has five different accessory proteins and SARS-CoVhas eight different accessory proteins, which share no sequence similarity. These differences, which presumably have different effects on induction and signaling of type 1 interferon, could explain why MERS-CoV is more sensitive to interferon than is SARS-CoV. This difference in interferon sensitivity has implications for treatment because type 1 interferon has been used to treat patients infected with SARS-CoV and MERS-CoV

MERS-CoV is distinct from SARS coronavirus and distinct from the common-cold coronavirus and known endemic human beta coronaviruses HCoV-OC43 and HCoV-HKU1. Until 23 May 2013, MERS-CoV had frequently been referred to as a SARS-like virus, or simply the novel coronavirus, and early it was referred to colloquially on message boards as the "Saudi SARS"

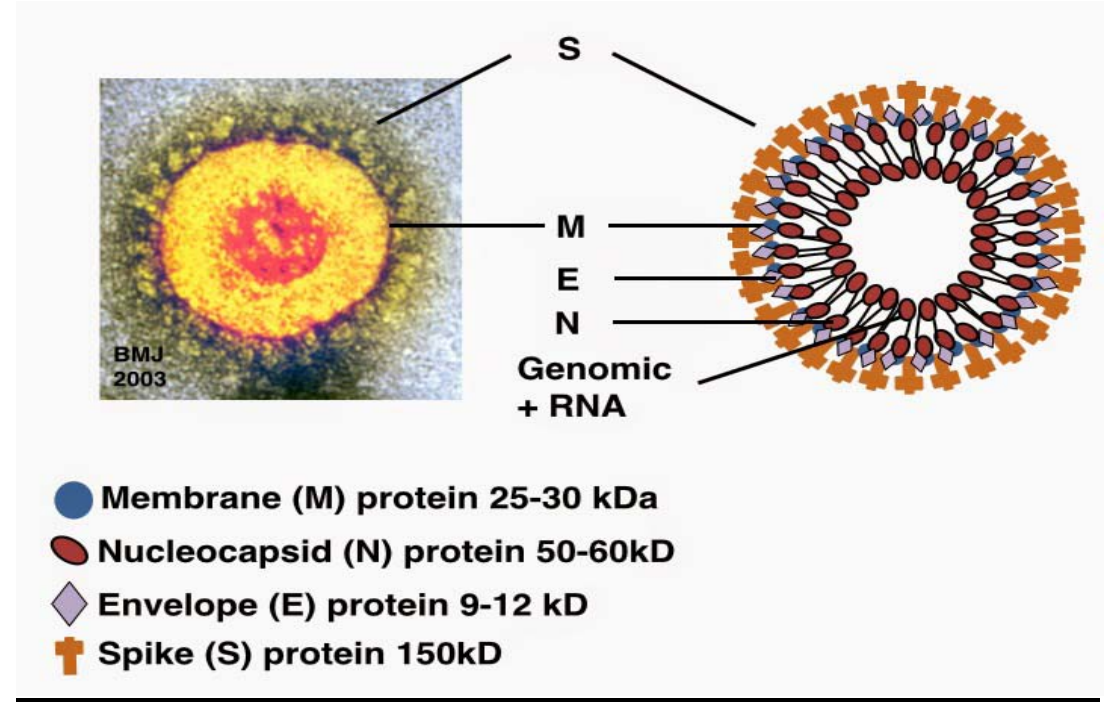

\section{Origin}

The first confirmed case was reported in Saudi Arabia 2012. Egyptian virologist Dr. Ali Mohamed Zaki isolated and identified a previously unknown coronavirus from the man's lungs. Dr. Zaki then posted his findings on 24 September 2012 on ProMED-mail. The isolated cells showed cytopathic effects (CPE), in the form of rounding and syncytia formation.

A second case was found in September 2012, a 49-year-old male living in Qatar presented with similar flu symptoms, and a sequence of the virus was nearly identical to that of the first case. In November 2012, similar cases appeared in Qatar and Saudi Arabia. Additional cases were noted, with deaths associated, and rapid research and monitoring of this novel coronavirus began.

It is not certain whether the infections are the result of a single zoonotic event with subsequent human-to-human transmission, or if the multiple geographic sites of infection represent multiple zoonotic events from a common unknown source. 


\section{Middle east respiratory syndrome 3-D image}

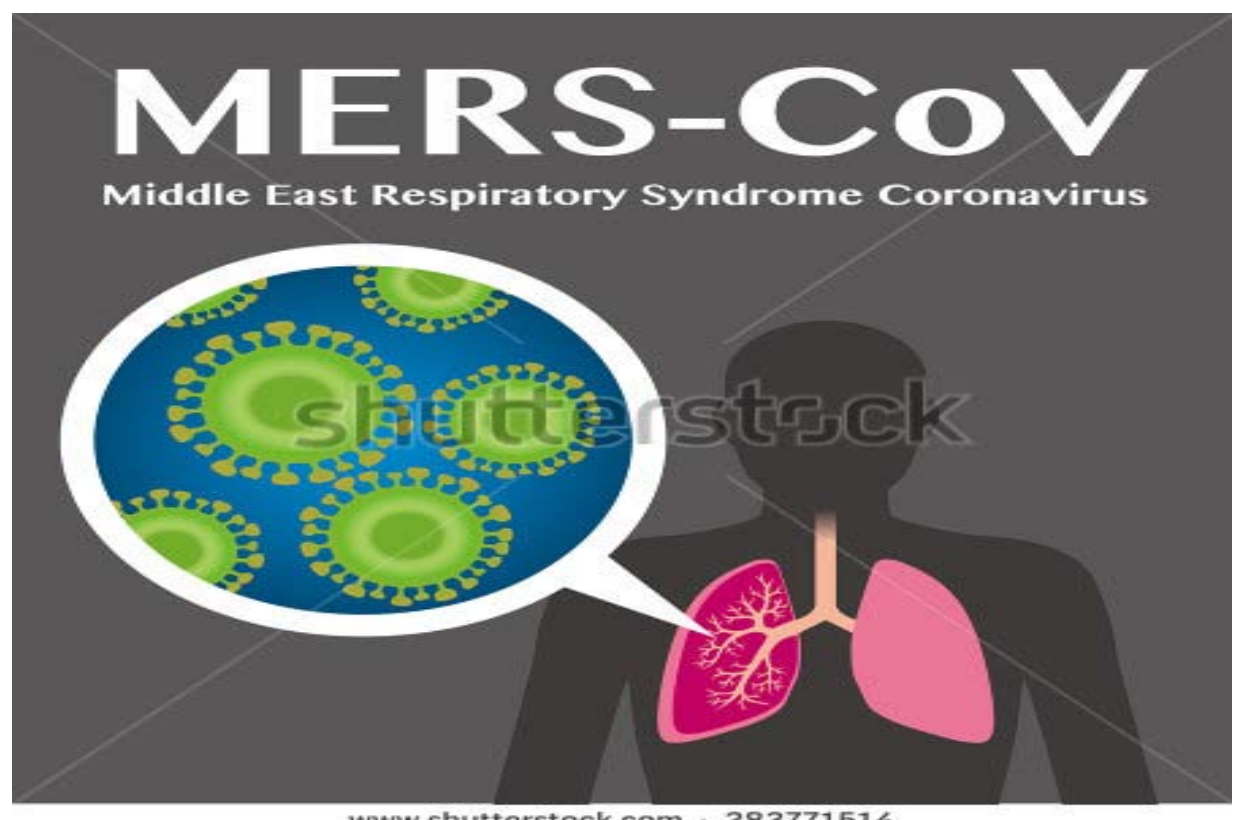

www.shutterstock.com - 283771514

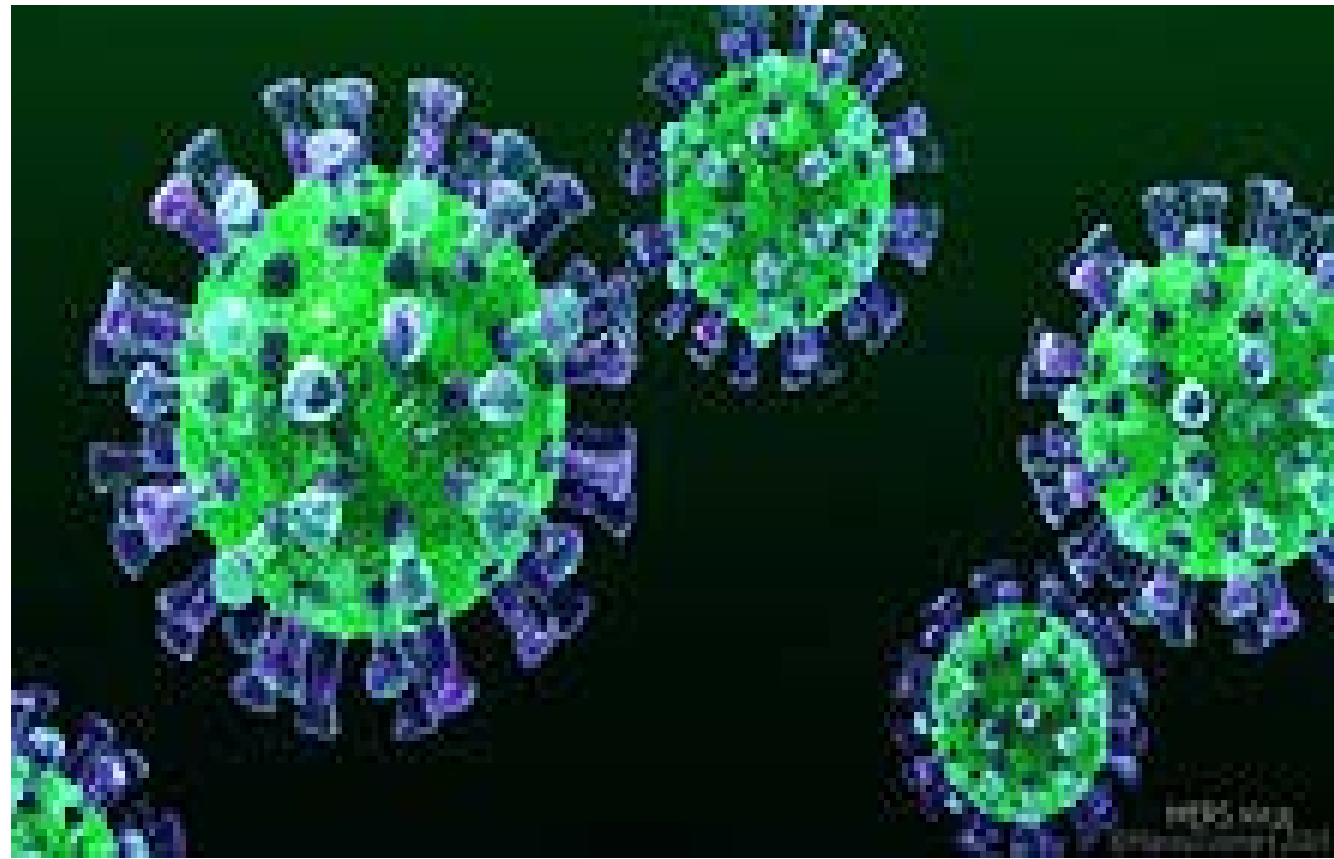

\section{Tropism}

In humans, the virus has a strong tropism for nonciliated bronchial epithelial cells, and it has been shown to effectively evade the innate immune responses and antagonize interferon (IFN) production in these cells. This tropism is unique in that most respiratory viruses target ciliated cells.

Due to the clinical similarity between MERS-CoV and SARS-CoV, it was proposed that they may use the same cellular receptor; the exopeptidase, angiotensin converting enzyme 2 (ACE2). However, it was later discovered that neutralization of ACE2 by recombinant antibodies does not prevent MERS-CoV infection. Further research identified dipeptyl peptidase 4 (DPP4; also known as CD26) as a functional cellular receptor for MERS-CoV. Unlike other known coronavirus receptors, the enzymatic activity of DPP4 is not required for infection. As would be expected, the amino acid 
sequence of DPP4 is highly conserved across species and is expressed in the human bronchial epithelium and kidneys. Bat DPP4 genes appear to have been subject to a high degree of adaptive evolution as a response to coronavirus infections, so the lineage leading to MERS-CoV may have circulated in bat populations for a long period of time before being transmitted to people.

\section{Transmission}

\section{How is Novel Coronavirus transmitted?}

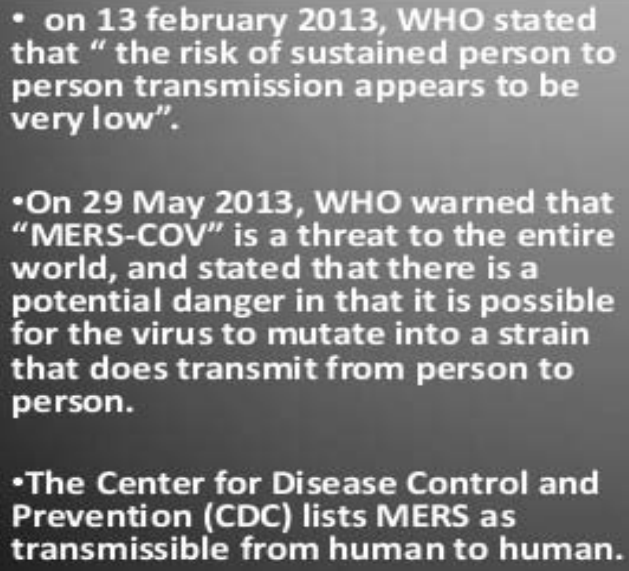

-The Center for Disease Control and Prevention (CDC) lists MERS as transmissible from human to human.

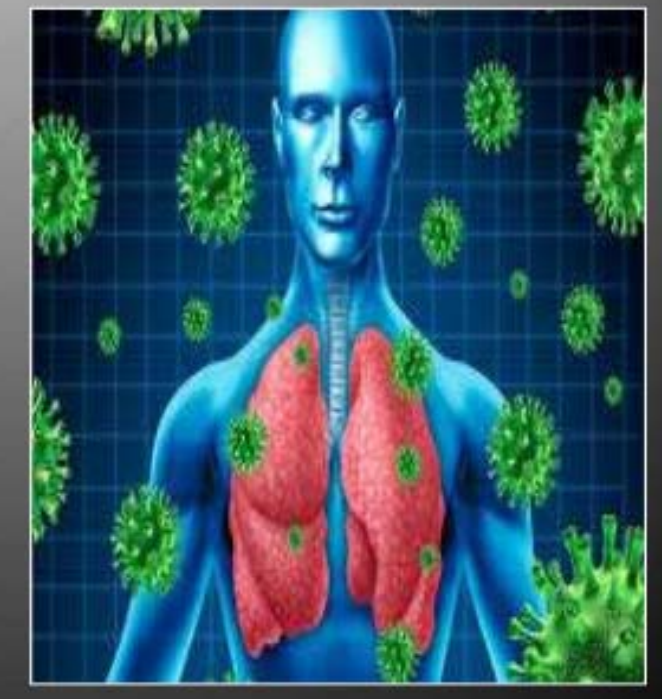

On 13 February 2013, the World Health Organization stated "the risk of sustained person-to-person transmission appears to be very low."The cells MERS-CoV infects in the lungs only account for 20\% of respiratory epithelial cells, so a large number of virions are likely needed to be inhaled to cause infection. The infection of healthcare workers (HCW) has led to concerns of human to human transmission.

Human-to-human transmission of MERS-CoV has been confirmed by epidemiological and genomic studies of cases associated with hospital and household MERS outbreaks. In a hospital-based outbreak that occurred in April and May, 2013, in Al-Hasa, an eastern province of Saudi Arabia, 23 patients receiving hemodialysis or in intensive care units were infected with a single clade of virus, with a mortality rate of $65 \%$. Spread was assumed largely to occur via large droplets and contact, although the possibility of airborne or fomite transmission was not excluded. Most infections resulted from person-to-person spread, emphasizing the importance of appropriate contact and droplet precautions to prevent transmission to other patients, health-care workers, and family members. Person-to-person spread in health-care facilities was also implicated in the $2014 \mathrm{MERS}-\mathrm{CoV}$ outbreak in Jeddah, Saudi Arabia. Patients that were initially considered primary cases were, on further investigation, found to have been exposed to MERS-CoV-infected patients, generally in health-care facilities.

MERS-CoV has spread from ill people to others through close contact, such as caring for or living with an infected person. Infected people have spread MERS-CoV to others in healthcare settings, such as hospitals. Researchers studying MERS have not seen any ongoing spreading of MERS-CoV in the community. 
MERS-CoVs isolated from single outbreaks are closely related. Genomic data are most consistent with human-to-human spread accompanied by periodic reintroduction of the virus into human populations. For MERS-CoV is generally estimated to be less than $0 \cdot 7$, substantially less than $1 \cdot 0$ associated with epidemic potential, making sustained transmission of the virus unlikely unless it mutates. By comparison, $\mathrm{R}_{\mathrm{o}}$ for SARS-CoV was more than, consistent with sustained transmission during the SARS epidemic

The Centers for Disease Control and Prevention (CDC) list MERS as transmissible from human-tohuman. MERS-CoV has been shown to spread between people who are in close contact. Transmission from infected patients to healthcare personnel has also been observed. Clusters of cases in several countries are being investigated.

On the 28th of May, the CDC revealed that the Illinois man who was originally thought to have been the first incidence of person to person spread (from the Indiana man at a business meeting), had in fact tested negative for MERS-CoV. After completing additional and more definitive tests using a neutralizing antibody assay, experts at the CDC have concluded that the Indiana patient did not spread the virus to the Illinois patient. Tests concluded that the Illinois man had not been previously infected. It is possible for silent MERS to occur, this is when the patient does not develop symptoms. Early research has shown that up to $20 \%$ of cases show no signs of active infection but have MERS-CoV antibodies in their blood.

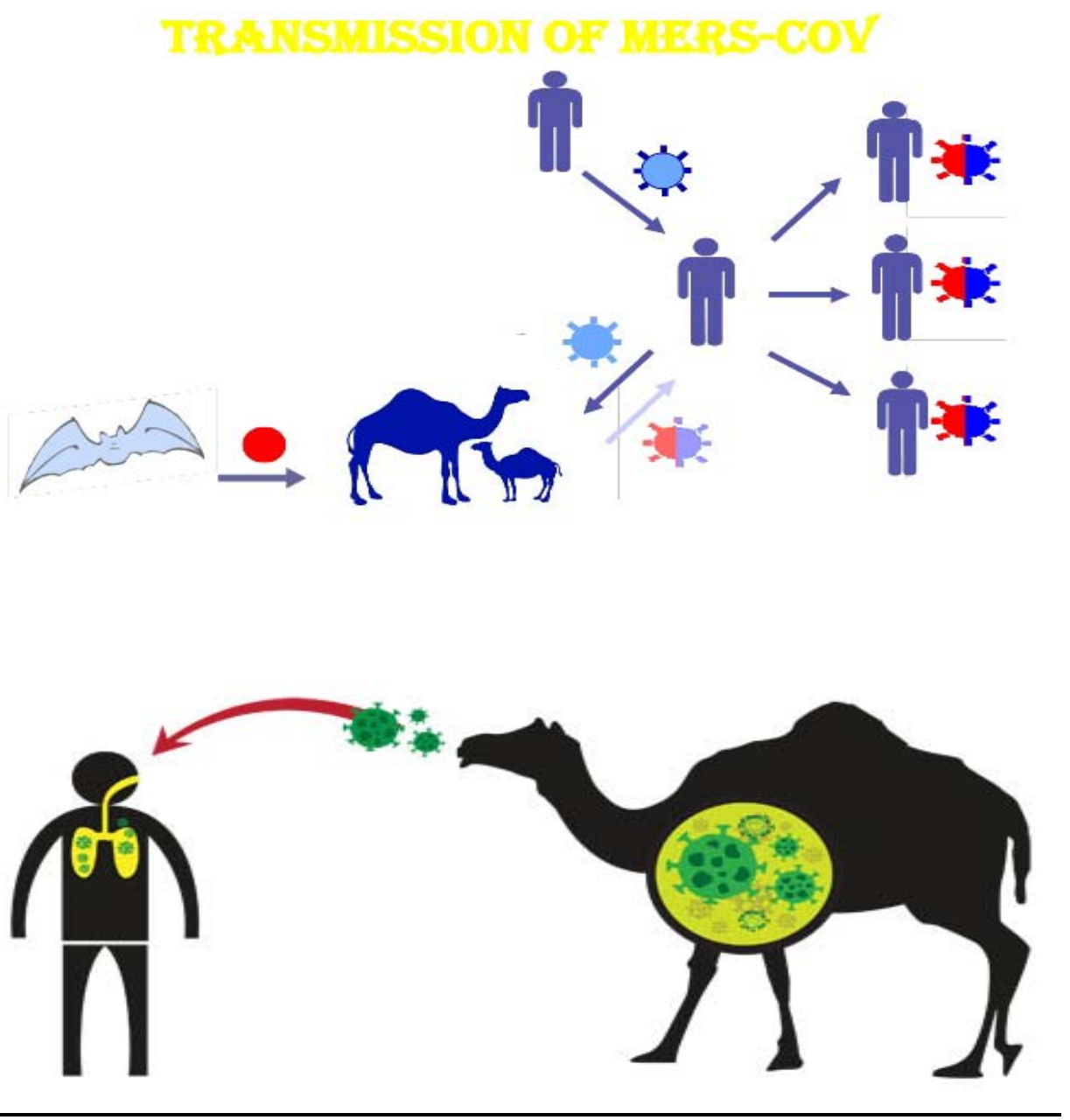




\section{Evolution}

The virus appears to have originated in bats. The virus itself has been isolated from a bat. This virus is closely related to the Tylonycteris bat coronavirus HKU4 and Pipistrellus bat coronavirus HKU5. Serological evidence shows that these viruses have infected camels for at least 20 years. The most recent common ancestor of several human strains has been dated to March 2012 (95\% confidence interval December 2011 to June 2012).

The evidence available to date suggests that the viruses have been present in bats for some time and had spread to camels by the mid-1990s. The viruses appear to have spread from camels to humans in the early 2010s. The original bat host species and the time of initial infection in this species has yet to be determined

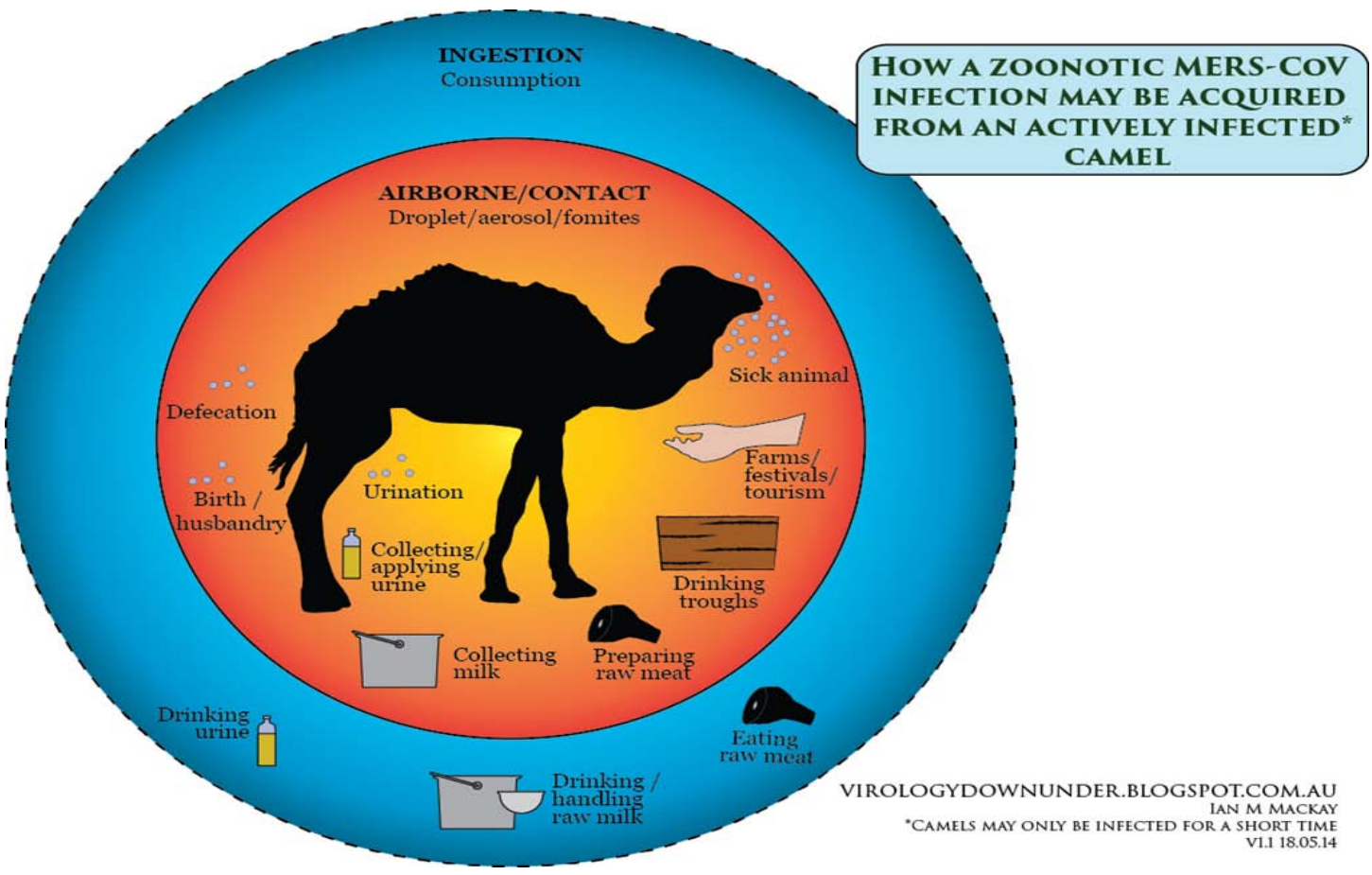

\section{Natural reservoir}

The virus is related to one found in the Egyptian tomb bat. In September 2012 Ron Fouchier speculated that the virus might have originated in bats. Work by epidemiologist Ian Lipkin of Columbia University in New York showed that the virus isolated from a bat looked to be a match to the virus found in humans. 2c beta coronaviruses were detected in Nycteris bats in Ghana and Pipistrellus bats in Europe that are phylogenetically related to the MERS-CoV virus.

The major natural reservoir where humans gets the virus infection remained unknown till on 9 August 2013, a report in the journal The Lancet Infectious Diseases showed that 50 out of 50 (100\%) blood serum from Omani camels and 15 of 105 (14\%) from Spanish camels had protein-specific antibodies against the MERS-CoV spike protein. Blood serum from European sheep, goats, cattle, and other camelids had no such antibodies.

On 5 September 2013 a seroepidemiological study published in the journal of Euro surveillance by R. A Perera et al where they investigated 1343 human and 625 animal sera indicated, the abundant presence of MERS-CoV specific antibody in 108 out of 110 Egyptian dromedary camels but not in other animals such as goats, cows or sheep in this region. These are the first and significant scientific reports that indicated the role of "dromedary camels" as a reservoir of MERS-CoV. At least one person who has fallen sick with MERS was known to have come into contact with camels or recently drank camel milk. 
Saudi Arabia and the United Arab Emirates produce and consume large amounts of camel meat. The possibility exists that African or Australian bats harbor the virus and transmit it to camels. Imported camels from these regions might have carried the virus to the Middle East.

In 2013 MERS-CoV was identified in three members of a dromedary camel herd held in a Qatar barn, which was linked to two confirmed human cases who have since recovered. The presence of MERS-CoV in the camels was confirmed by the National Institute of Public Health and Environment (RIVM) of the Ministry of Health and the Erasmus Medical Center (WHO Collaborating Center), the Netherlands. None of the camels showed any sign of disease when the samples were collected. The Qatar Supreme Council of Health advised in November 2013 that people with underlying health conditions, such as heart disease, diabetes, kidney disease, respiratory disease, the immunosuppressed, and the elderly, avoid any close animal contacts when visiting farms and markets, and to practice good hygiene, such as washing hands.

According to the 27 March 2014 MERS-CoV summary update, recent studies support that camels serve as the primary source of the MERS-CoV infecting humans, while bats may be the ultimate reservoir of the virus. Evidence includes the frequency with which the virus has been found in camels to which human cases have been exposed, seriological data which shows widespread transmission in camels, and the similarity of the camel CoV to the human CoV.

\section{Taxonomy}

MERS-CoV is more closely related to the bat coronaviruses HKU4 and HKU5 (lineage 2C) than it is to SARS-CoV (lineage 2B) more than $90 \%$ sequence identity with their closest relationships, bat coronaviruses HKU4 and HKU5 and therefore considered to belong to the same species by the International Committee on Taxonomy of Viruses (ICTV).

Mnemonic:

Taxon identifier:

Scientific name: Middle East respiratory syndrome coronavirus

Common name: MERS-CoV

Synonym: Severe acute respiratory syndrome coronavirus

Other names:

Novel coronavirus (nCoV)

\section{MERS-CoV}

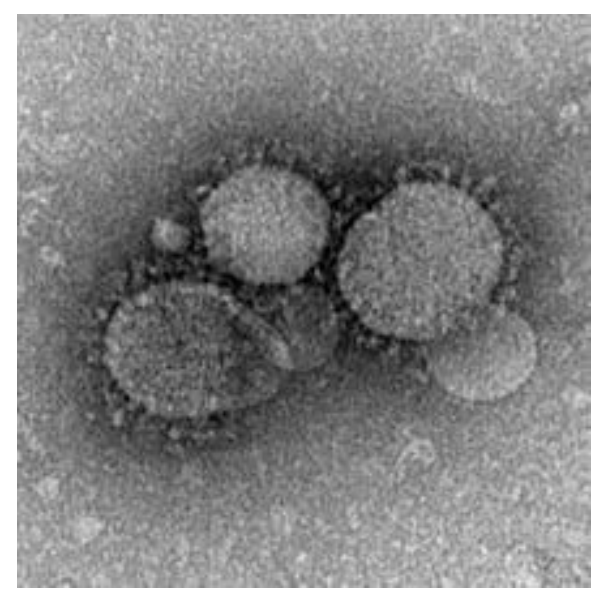

MERS-CoV particles as seen by negative stain electron microscopy. Virions contain characteristic club-like projections emanating from the viral membrane. 


\begin{tabular}{|l|l|}
\hline Virus classification \\
\hline Group: & Group IV ((+)ssRNA) \\
\hline Order: & Nidovirales \\
\hline Family: & Coronaviridae \\
\hline Subfamily: & Coronavirinae \\
\hline Genus: & Betacoronavirus \\
\hline Species: & MERS-CoV \\
\hline
\end{tabular}

\section{Modes of transmission}

According to the limited information available so far, there is no accurate scientific evidence identifying the way through which the virus can be transmitted from one person to another. But, it is likely to be similar to the other kinds of Coronavirus. $\mathrm{MOH}$ is making every effort, in collaboration with its partners in the international organizations, including the WHO, to learn more about ways of transmission, including through animals. The ways of human-to-human Corona transmission include the following:

1. Direct transmission through droplets produced during coughing or sneezing.

2. Indirect transmission through touching surfaces and devices contaminated with the virus, and then touching the mouth, nose or eyes.

3. Direct contact with infected patients.

4. Put on face-masks only if you are sick or visiting sick patients.

Possible sources and modes of transmission - It seems likely that dromedary camels are the primary animal host for MERS-CoV. The presence of case clusters strongly suggests that human-tohuman transmission occurs

Serologic studies have shown low prevalence of MERS-CoV antibodies in humans in Saudi Arabia. A broad antibody survey of 10,009 individuals representative of the general population of Saudi Arabia found seropositivity in 15 (0.15 percent), all but one of whom resided in five interior provinces (of 13 total provinces). In a separate survey included in the same report, 87 camel shepherds and 140 slaughterhouse workers were tested, of whom (3.1 percent) were seropositive.

Among 5235 adult pilgrims from 22 countries who visited Mecca, Saudi Arabia, for Hajj in 2013, none had a positive MERS-CoV polymerase chain reaction (PCR) from the nasopharynx; 3210 individuals were screened pre-Hajj, and 2025 were screened post-Hajj

Bats- Studies performed in Europe, Africa, and Asia, including the Middle East, have shown that coronavirus RNA sequences are found frequently in bat fecal samples and that some of these sequences are closely related to MERS-CoV sequences I n a study from Saudi Arabia, 823 fecal and rectal swab samples were collected from bats, and, using a PCR assay, many coronavirus sequences were found. Most were unrelated to MERS-CoV, but, notably, one 190 nucleotide sequence in the RNA-dependent RNA polymerase (RdRp) gene was amplified that had 100 percent identity with a MERS-CoV isolate cloned from the index patient with MERS-CoV infection; the sequence was detected from a fecal pellet of a Taphozousperforatus bat captured from a site near the home of the patient. MERS-CoV grows readily in several bat-derived cell lines.

Although bats might be a reservoir of MERS-CoV, it is unlikely that they are the immediate source for most human cases because human contact with bats is uncommon.

Camels - As noted above, it is likely that camels serve as hosts for MERS-CoV. The strongest evidence of camel-to-human transmission of MERS-CoV comes from a study in Saudi Arabia in which MERS-CoV was isolated from a man with fatal infection and from one of his camels; fullgenome sequencing demonstrated that the viruses isolated from the man and his camel were identical. The study had the following findings: 
Texila International Journal of Public Health

Volume 4, Issue 4, Dec 2016

\section{Clinical features}

The clinical manifestations of MERS-CoV infection range from asymptomatic infection to severe pneumonia with acute respiratory distress syndrome, septic shock, and multiorgan failure resulting in death.

On the basis of data related to human-to-human transmission in several clusters, the incubation period has been estimated as more than 5 days, but could be as long as 2 weeks. Median time from onset of symptoms to hospital admission is $4 \cdot 0$ days (range $0-16, n=62$ ), from onset of symptoms to admission to an intensive care unit is $5 \cdot 0(1-15, n=35)$, and from onset of symptoms to death is 11.5 days

Most people confirmed to have MERS-CoV infection have had severe acute respiratory illness with symptoms of:

- fever

- cough

- shortness of breath

Some people also had gastrointestinal symptoms including diarrhea and nausea/vomiting. For many people with MERS, more severe complications followed, such as pneumonia and kidney failure. About 3-4 out of every 10 people reported with MERS have died. Most of the people who died had an underlying medical condition. Some infected people had mild symptoms (such as coldlike symptoms) or no symptoms at all; they recovered.

People with pre-existing medical conditions (also called comorbidities) may be more likely to become infected with MERS-CoV, or have a severe case. Pre-existing conditions from reported cases for which we have information have included diabetes; cancer; and chronic lung, heart, and kidney disease. Individuals with weakened immune systems are also at higher risk for getting MERS or having a severe case.

Based on information we have to date, the incubation period for MERS (time between when a person is exposed to MERS-CoV and when they start to have symptoms) is usually about 5 or 6 days, but can range from 2-14 days.

MERS typically begins with fever, cough, chills, sore throat, myalgia, and arthralgia, followed by dyspnoea and rapid progression to pneumonia within the first week, often requiring ventilatory and other organ support. Although most patients with symptomatic disease present with respiratory illness, immunocompromised patients can present with fever, chills, and diarrhea and later develop pneumonia. Similar to SARS, at least a third of patients with MERS have gastrointestinal symptoms, such as vomiting and diarrhea. Risk factors for development of severe disease, in addition to an immunocompromised state, include comorbidity (eg, obesity, diabetes, cardiac disease, and lung disease). Concomitant infections and low albumin concentration are predictors of severe illness, and age older than 65 years was associated with mortality in a case series in Saudi Arabia. The few data available about viral dynamics and clinical course suggest that patients with MERS have a shorter time from illness onset to clinical presentation and to a requirement for ventilatory support than do patients with SARS and higher respiratory tract viral loads during the first week of the illness.

SARS and other severe viral illnesses, common laboratory findings of MERS include leucopenia, particularly lymphopenia. Some patients have a consumptive coagulopathy and high creatinine, lactate dehydrogenase, and liver enzyme concentrations. Co-infection with other respiratory viruses (eg, parainfluenza, rhinovirus, influenza A virus [H1N1], herpes simplex virus, influenza B virus) has been reported and nosocomial bacterial infections (including Klebsiellapneumoniae, Staphylococcus aureus, Acinetobacter species, Candida species) have occurred in patients receiving invasive mechanical ventilation.

Chest radiography and tomography findings of MERS are consistent with viral pneumonitis and acute respiratory distress syndrome, with bilateral hilar infiltration, unilateral or bilateral patchy densities or infiltrates, segmented or lobar opacities, ground-glass opacities, and small pleural 
effusions in some cases. Lower lobes are generally affected more than are upper lobes early in the course of illness with more rapid radiographic progression than in SARS.

Some patients have viral RNA in blood, urine, and stool but at much lower viral loads than in the respiratory tract.95 MERS-CoV viral loads and genome fractions in upper respiratory tract specimens (eg, nasopharyngeal swabs) are lower than in lower respiratory tract specimens, such as tracheal aspirates and bronchoalveolar lavage fluid, which probably accounts for the inefficiency of interhuman transmission. Lower respiratory tract excretion of MERS-CoV RNA can be detected after 1 month of illness in most patients, suggesting that prolonged shedding could be a source for spread in outbreaks.

\section{Signs and symptoms of Anaphylaxis}

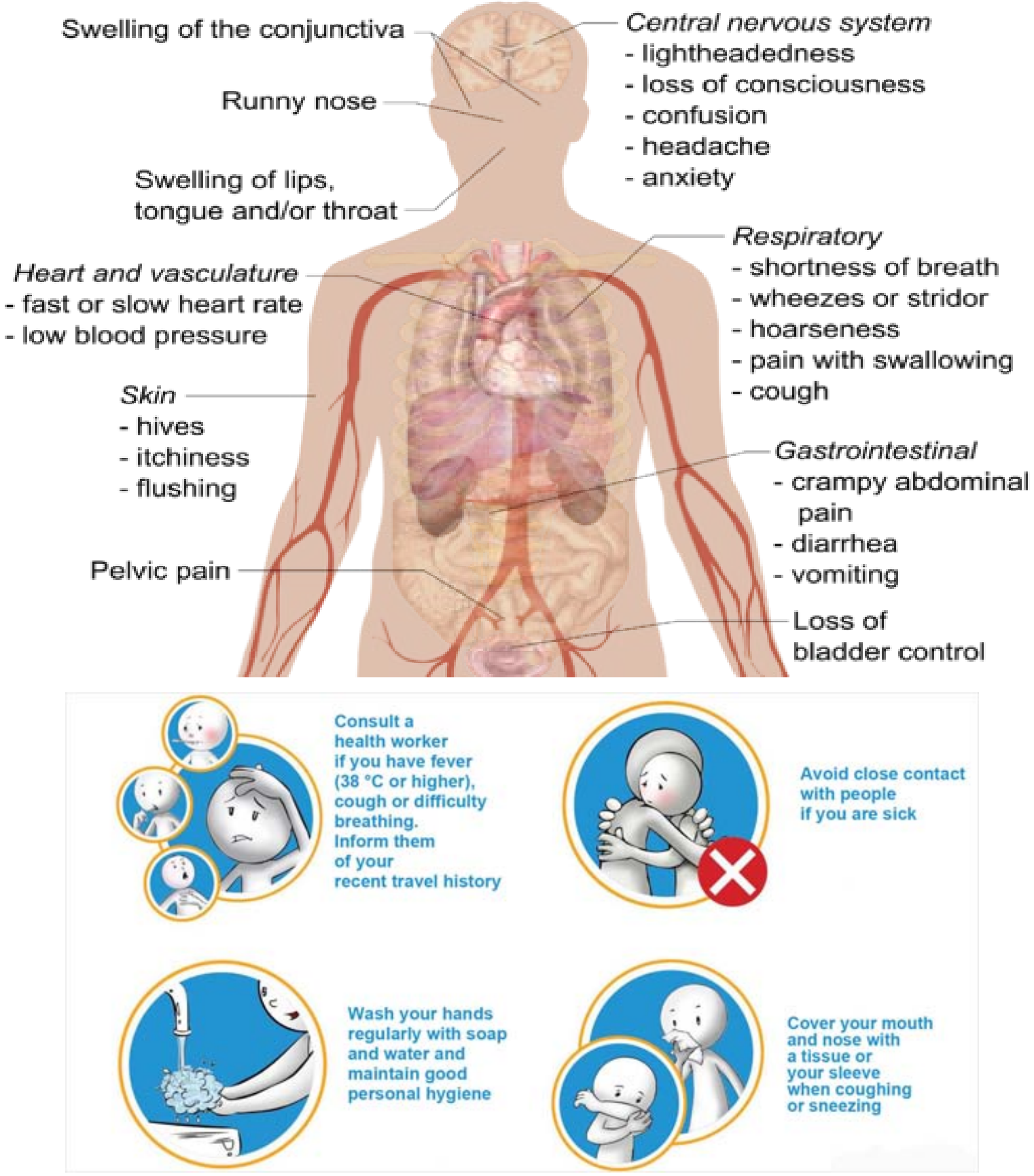


Texila International Journal of Public Health

Volume 4, Issue 4, Dec 2016

\section{Diagnosis}

Because lower respiratory tract specimens such as bronchoalveolar lavage fluid, sputum, and tracheal aspirates contain the highest viral loads, they should be collected whenever possible. MERS can be confirmed by detection of viral nucleic acid or by serology. The presence of viral nucleic acid can be confirmed either by positive real-time reverse transcription PCR on at least two specific genomic targets or by a single positive target with sequencing of a second positive PCR product. Available real-time reverse transcription PCR tests include an assay targeting RNA upstream of the E gene (upE) and assays targeting open reading frames $1 \mathrm{~b}(O R F 1 b)$ and $1 \mathrm{a}(O R F 1 a)$. The assay for the upE target is highly sensitive and is recommended for screening; the ORF $1 a$ assay is of equal sensitivity. The ORF $1 b$ assay is less sensitive but is useful for confirmation. These assays have not shown cross-reactivity with other respiratory viruses including human coronaviruses. Two target sites on the MERS-CoV genome suitable for sequencing to aid confirmation are in the RNA-dependent RNA polymerase ( $R d R p$; present in $O R F 1 b$ ) and $N$ genes

In MERS cases confirmed by PCR, serial sampling for PCR testing from the upper and lower respiratory tracts and other body parts (eg, serum, urine, and stool) are recommended to understand viral replication kinetics and to guide infection control. Respiratory samples should be collected at least every 2-4 days to confirm viral clearance after two consecutive negative results are obtained.

For confirmation of infection by antibody detection, paired serum samples should be collected 1421 days apart with the first taken during the first week of illness. A positive screening assay (ELISA, immunofluorescence assay) should be followed by a confirmatory (neutralisation) assay. Single samples might also be valuable for identification of probable cases and should be collected at least 14 days after the onset of symptoms. Serological results should be carefully interpreted because they might be confounded by cross-reactivity against other coronaviruses

\section{Treatment}

No specific drug treatment exists for MERS and supportive treatment is the mainstay of management. Evidence-based recommendations for treatment provide the basis for decision making in clinical settings

MERS-CoV is readily inhibited by type 1 interferons (IFN- $\alpha$ and especially IFN- $\beta$ ) in cultured cells, and IFN- $\alpha 2 b$ combined with ribavirin can lessen lung injury and reduce lung titres when administered to rhesus macaques within $8 \mathrm{~h}$ of virus inoculation.

This combination was tested in severely ill patients, showing an improvement in survival at 14 days but not 28 days, possibly a result of administration in the advanced stages of disease

Several drugs inhibit MERS-CoV in cell culture, including ciclosporin and mycophenolic acid. Other compounds (chloroquine, chlorpromazine, loperamide, and lopinavir) inhibit virus replication (effective concentration50 3-8 $4 \mathrm{~mol} / \mathrm{L}$ ) in vitro, although whether these drugs will be useful in patients is unknown. MERS-CoV-specific peptide fusion inhibitors, which function similarly to the HIV drug enfuvirtide, diminish virus replication in cultured cells, providing a novel approach to MERS treatment.

\section{Prevention}

- Recommendations for prevention of MERS are available from WHO, the US Centers for Disease Control and Prevention, and the Saudi Ministry of Health. The main infection prevention and control measures are droplet precautions (wearing a surgical mask within $1 \mathrm{~m}$ of patients) and contact precautions (wearing gown and gloves on entering patients' rooms and removing them on leaving). Droplet precautions should be added to the standard precautions when providing care to all patients with signs of acute respiratory infection. Eye protection should be used when health-care workers care for probable or confirmed patients.

- Public Health England, US Centers for Disease Control and Prevention, and Saudi Ministry of Health recommendations for management of known or suspected MERS-CoV infection include 
the use of personal protective equipment such as gowns, gloves, eye protection (goggles or face shield), and respiratory protection equivalent to a fit-tested National Institute for Occupational Safety and Health-certified disposable N95 filtering face piece respirator. Patients with MERS should be placed in negative pressure rooms or in rooms in which room exhaust is filtered through high-efficiency particulate air filters. Airborne precautions with at least six air changes per hour should be applied in treatment rooms when performing aerosol-generating procedures. These recommendations are evidence-based and have proven to be effective in hospitals in affected countries.

- Camels infected with MERS-CoV can develop rhinitis or show no signs of infection and might shed virus through nasal and eye discharge and faeces. The virus can also be found in raw milk from infected camels. MERS-CoV is stable in camel breast milk for extended periods of time; thus, pasteurization or cooking is recommended to destroy the virus.

- Raw urine should not be used for medicinal purposes. Because signs of disease are non-specific, it is not possible to know whether an animal in a farm, market, race track, or slaughterhouse is excreting MERS-CoV without virological testing.

- Camel farm workers, slaughterhouse workers, market workers, veterinarians, and those handling camels at racing facilities should practice good personal hygiene, including frequent hand washing after touching animals, avoiding touching eyes, nose, or mouth with hands, and avoiding contact with sick animals. Consideration should also be given to wearing protective gowns and gloves while handling animals, especially if camels have signs of upper respiratory tract disease.

\section{Imaging findings at presentation in Saudi patients with Middle East respiratory syndrome coronavirus infection}
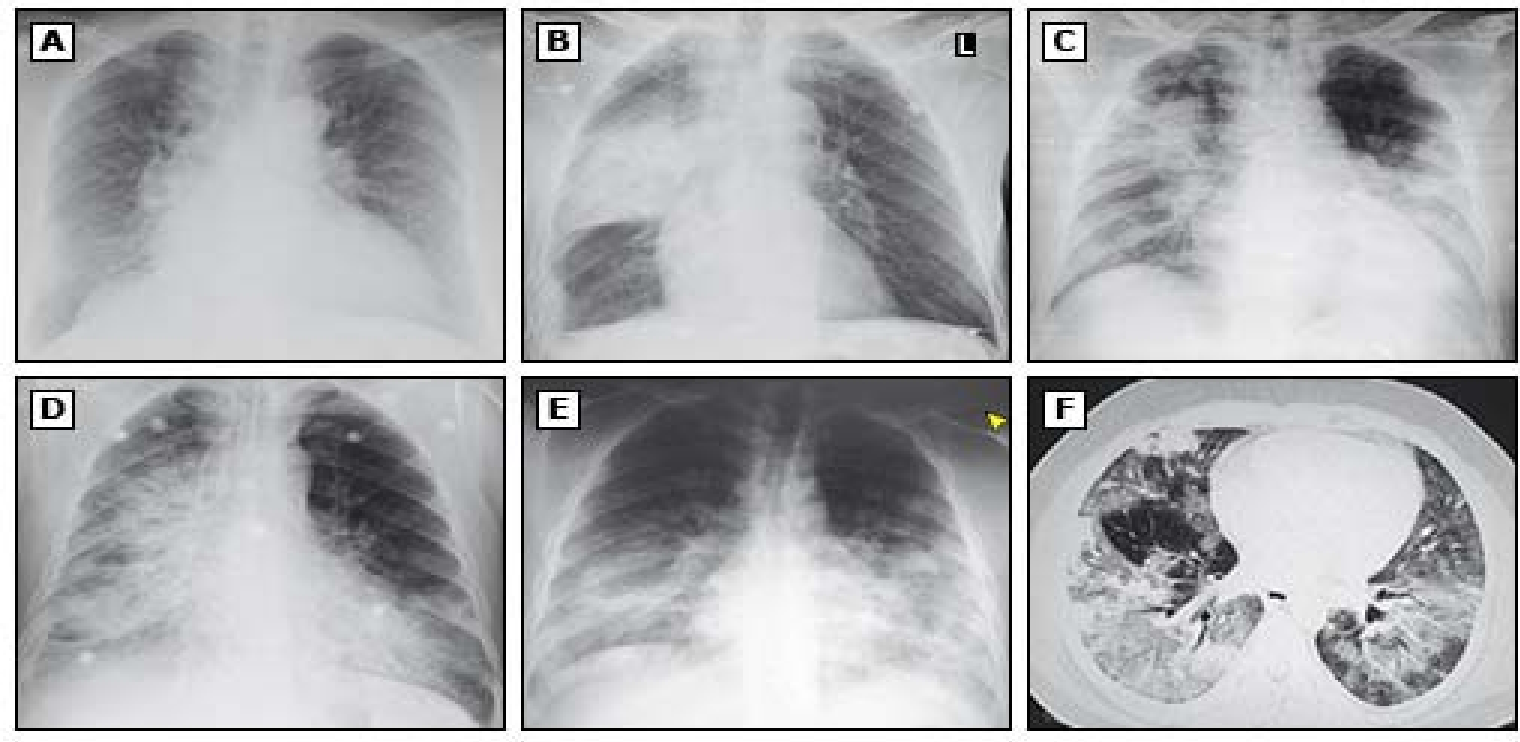

(Panel A) Chest radiograph of a 61-year-old man, showing bilateral fine reticulonodular air-space opacities, increased vascular markings, and cardiomegaly.

(Panel B) Chest radiograph of an 83-year-old man, showing right lung consolidation, right basal pleural thickening, and reticulonodular air-space opacities; rib fractures on the right are old.

(Panel C) Chest radiograph of a 56-year-old man, showing extensive bilateral extensive diffuse and focal alveolar space opacities, with opacification of the left lower lobe.

(Panel D) Chest radiograph of a 67-year-old man, showing extensive bilateral disease, with diffuse alveolar space densities, opacification, reticulonodular opacities, and bronchial wall thickening. 
(Panel E) Chest radiograph of a 49-year-old man, showing extensive bilateral mid and lower zone disease, with diffuse reticulonodular alveolar space opacities. A thoracic computed tomography scan in the same patient (Panel F) shows extensive bilateral opacities and ground-glass reticulonodular shadowing and bronchiolar wall thickening.

\section{Ways of protection against the Middle East Respiratory Syndrome-Coronavirus (MERC-CoV)}

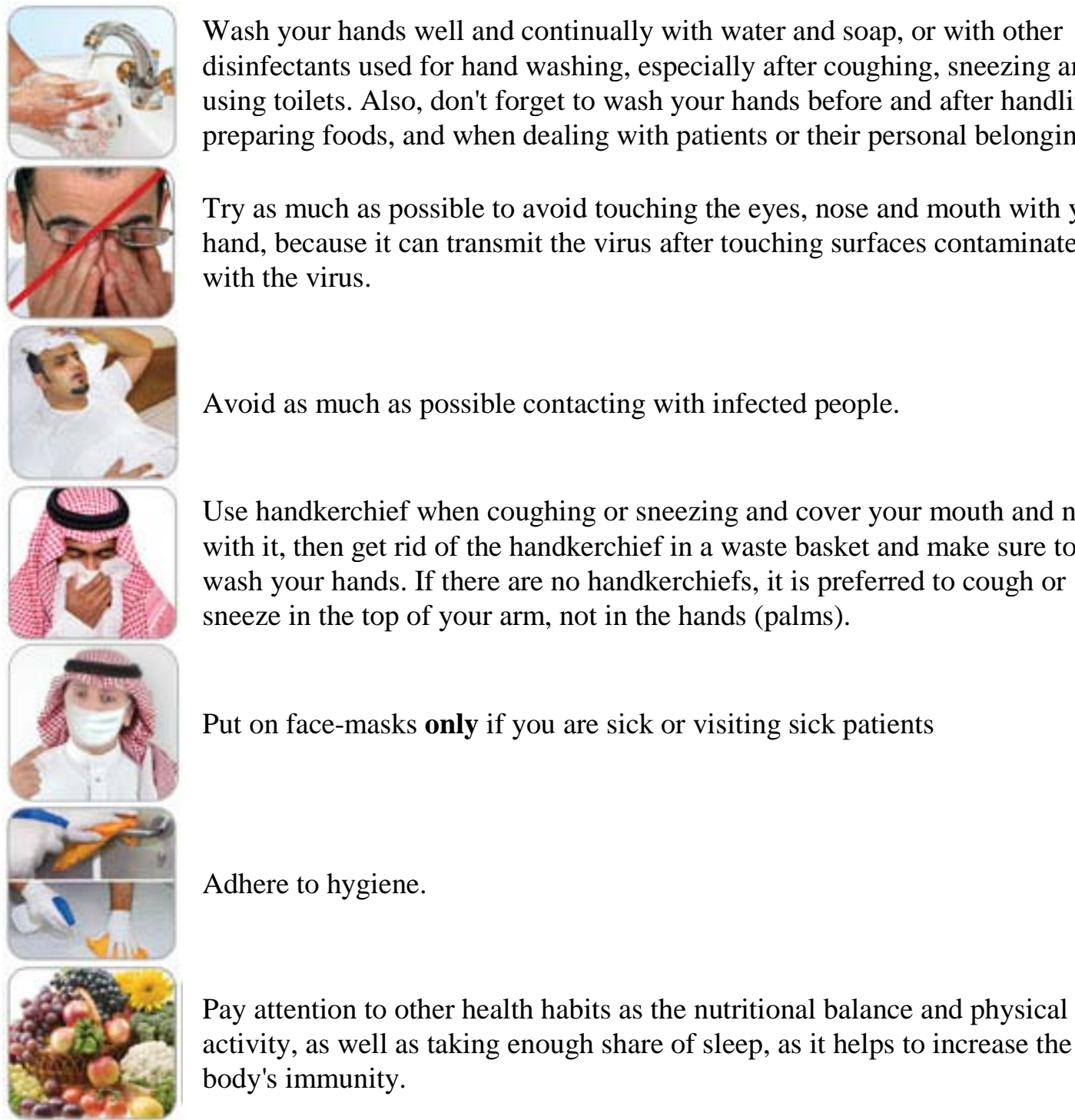

\section{Infection prevention and control recommendations for hospitalized patients with (MERS-CoV)}

Center for Disease Control (CDC) works 24/7 to protect people's health. It is the job of CDC to be concerned and move quickly whenever there is a potential public health problem. CDC continues to closely monitor the MERS situation globally. CDC is working with the World Health Organization and other partners to better understand the virus, how it spreads, the source, and risks to the public's health. We recognize the potential for MERS-CoV to spread further and cause more cases in the United States and globally. In preparation for this, we have:

- Continued to collaborate with international partners on epidemiologic and laboratory studies to better understand MERS 
- Improved the way we collect data about MERS cases

- Increased lab testing capacity in states to detect cases

- Developed guidance and tools for health departments to conduct public health investigations when MERS cases are suspected or confirmed

- Provided recommendations for healthcare infection control and other measures to prevent disease spread

- Provided guidance for flight crews, Emergency Medical Service (EMS) units at airports, and U.S. Customs and Border Protection (CPB) officers about reporting ill travelers to CDC

- Disseminated up-to-date information to the general public, international travelers, and public health partners

\section{Recommendations}

Definition of Healthcare Personnel (HCP) -HCP refers to all persons, paid and unpaid, working in healthcare settings whose activities potentially place them at risk for exposures to a patient with MERS-CoV.

\section{Minimize chance for exposures}

Ensure facility policies and practices are in place to minimize exposures to respiratory pathogens including MERS-CoV. Measures should be implemented before patient arrival, upon arrival, and throughout the duration of the affected patient's presence in the healthcare setting.

\subsection{Before arrival}

- When scheduling appointments, instruct patients and persons who accompany them to call ahead or inform HCP upon arrival if they have symptoms of any respiratory infection (e.g., cough, runny nose, fever ${ }^{1}$ ) and to take appropriate preventive actions (e.g., wear a facemask upon entry to contain cough, follow triage procedure).

\subsection{Upon arrival and during the visit}

- Take steps to ensure all persons with symptoms of a respiratory infection adhere to respiratory hygiene and cough etiquette, hand hygiene, and triage procedures throughout the duration of the visit. Consider posting visual alerts (e.g., signs, posters) at the entrance and in strategic places (e.g., waiting areas, elevators, cafeterias) to provide patients and HCP with instructions (in appropriate languages) about hand hygiene, respiratory hygiene, and cough etiquette. Instructions should include how to use facemasks (See definition of facemask in Appendix) or tissues to cover nose and mouth when coughing or sneezing, to dispose of tissues and contaminated items in waste receptacles, and how and when to perform hand hygiene.

- Provide space and encourage persons with symptoms of respiratory infections to sit as far away from others as possible. If available, facilities may wish to place these patients in a separate area while waiting for care.

- Ensure rapid triage and isolation of patients who might have MERS-CoV infection

- Identify patients at risk for having MERS-CoV infection before or immediately upon arrival to the hospital

- Implement triage procedures to detect patients at risk for having MERS-CoV infections during or before patient triage or registration (e.g., at the time of patient check-in) and ensure that all patients are asked about the presence of symptoms of a respiratory infection and history of travel to areas experiencing transmission of MERS-CoV or contact with possible MERS-CoVpatients. See the Interim Guidance for Healthcare Professionals for which patients to evaluate for MERSCoV.

- Immediately isolate those identified as at risk for having MERS-CoV infection

- Implement Respiratory Hygiene and Cough Etiquette (i.e., placing a facemask over the patient's nose and mouth) and isolate those at risk for MERS-CoV infection in an Airborne Infection 
Isolation Room (AIIR). See recommendations for "Patient Placement" below. Additional guidance for evaluating patients in U.S. for MERS-CoV infection can be found at the CDC Middle East Respiratory Syndrome (MERS) website.

- Provide supplies to perform hand hygiene to all patients upon arrival to facility (e.g., at entrances of facility, waiting rooms, at patient check-in) and throughout the entire duration of the visit to the healthcare setting.

\section{Ensure adherence to standard, contact and airborne precautions}

Standard precautions assume that every person is potentially infected or colonized with a pathogen that could be transmitted in the healthcare setting. Elements of standard precautions that apply to patients with respiratory infections, including those caused by MERS-CoV, are summarized below. Attention should be paid to training and proper donning, doffing and disposal of any personal protective equipment. who enter the room of a patient with suspected or confirmed MERS-CoV should adhere to Standard, Contact, and Airborne precautions, including the following:

\subsection{Hand hygiene}

- HCP should perform hand hygiene before and after all patient contact, contact with potentially infectious material, and before putting on and upon removal of PPE, including gloves. Hand hygiene in healthcare settings can be performed by washing with soap and water or using alcohol-based hand rubs. If hands are visibly soiled, use soap and water, not alcohol-based hand rubs.

- Healthcare facilities should ensure that facilities and supplies for performing hand hygiene are readily available to all personnel.

\subsection{Personal protective equipment}

- Employers should select appropriate PPE and provide it to workers in accordance with OSHA's PPE standards (29 CFR 1910 Subpart I). Workers must receive training on and demonstrate an understanding of when to use PPE; what PPE is necessary; how to properly don (put on), use, doff (take off) PPE; how to properly dispose of or disinfect and maintain PPE; and the limitations of PPE. Any reusable PPE must be properly cleaned, decontaminated, and maintained after and between uses.

\subsection{Gloves}

- Put on clean, non-sterile gloves upon entry into the patient room or care area. Change gloves if they become torn or heavily contaminated.

- Remove and discard gloves immediately upon leaving the patient room or care area. Please see section below on "Using More than one Kind of Personal Protective Equipment (PPE)" for recommended sequence of PPE removal.

\subsection{Gowns}

- Put on a clean disposable gown upon entry into the patient room or area. Change the gown if it becomes soiled. Remove and discard the gown immediately upon leaving the patient room or care area.

\subsection{Respiratory protection}

- Use respiratory protection (i.e., a respirator) that is at least as protective as a fit-tested NIOSHcertified disposable N95 filtering facepiece respirator upon entry to the patient room or care area. See appendix for respirator definition.

- The respirator should be the last part of the PPE ensemble to be removed. If reusable respirators are used, they must be cleaned and disinfected according to manufacturer's reprocessing 
instructions prior to re-use. If disposable respirators are used, they should be removed and discarded after leaving the patient room or care area and closing the door.

- Respirator use must be in the context of a complete respiratory protection program in accordance with Occupational Safety and Health Administration (OSHA) Respiratory Protection standard Staff should be medically cleared and fit-tested if using respirators with tight-fitting facepieces (e.g disposable N95) and trained in the proper use of respirators, safe removal and disposal, and medical contraindications to respirator use.

\subsection{Eye Protection}

- Put on eye protection (e.g., a disposable face shield) upon entry to the patient room or care area. Remove and discard eye protection immediately upon leaving the patient room or care area. Reusable eye protection (e.g., goggles) must be cleaned and disinfected according to manufacturer's reprocessing instructions prior to re-use.

\subsection{Using more than one kind of personal protective equipment (PPE)}

- Different types of PPE are used together to prevent multiple routes of transmission.

- The following sequence is a general approach to putting on this PPE combination for respiratory pathogens: first gown; then respirator; then goggles or face shield; then gloves.

- The following sequence is a general approach to removing PPE for respiratory pathogens: first gloves; then goggles or face shield; then gown; then respirator.

- Except for respirator, remove PPE at doorway or in anteroom. Remove respirator after leaving patient room and closing door.

- Careful attention should be given to prevent contamination of clothing and skin during the process of removing PPE.

- Perform hand hygiene as described above immediately before putting on and after removing all PPE.

\subsection{Patient placement}

- Place a patient who might be infected with MERS-CoV in an Airborne Infection Isolation Room (AIIR) that has been constructed and maintained in accordance with current guidelines.

- AIIRs are single patient rooms at negative pressure relative to the surrounding areas, and with a minimum of 6 air changes per hour (12 air changes per hour are recommended for new construction or renovation). Air from these rooms should be exhausted directly to the outside or be filtered through a high-efficiency particulate air (HEPA) filter before recirculation. Room doors should be kept closed except when entering or leaving the room, and entry and exit should be minimized. Facilities should monitor and document the proper negative-pressure function of these rooms.

- If an AIIR is not available, the patient should be transferred as soon as is feasible to a facility where an AIIR is available. Pending transfer, place a facemask on the patient and isolate him/her in an examination room with the door closed. The patient should not be placed in any room where room exhaust is recirculated without high-efficiency particulate air (HEPA) filtration.

- Once in an AIIR, the patient's facemask may be removed; the facemask should remain on if the patient is not in an AIIR. Limit transport and movement of the patient outside of the AIIR to medically-essential purposes. When outside of the AIIR, patients should wear a facemask to contain secretions.

- Only essential personnel should enter the AIIR. Implement staffing policies to minimize the number of HCP who enter the room.

- Facilities should consider caring for these patients with dedicated HCP to minimize risk of transmission and exposure to other patients and other HCP.

- Facilities should keep a log of all persons who care for OR enter the rooms or care area of these patients. 
- Once the patient vacates a room, unprotected individuals, including HCP, should not be allowed in that room until sufficient time has elapsed for enough air changes to remove potentially infectious particles. More information on clearance is available. In addition, the room should undergo appropriate cleaning and surface disinfection before unprotected individuals are allowed to reenter it.

\section{Manage visitor access and movement within the facility}

1. Establish procedures for monitoring, managing and training visitors.

2. All visitors should follow respiratory hygiene and cough etiquette precautions while in the common areas of the facility.

3. Restrict visitors from entering the MERS-CoV patient's room. Facilities can consider exceptions based on end-of-life situations or when a visitor is essential for the patient's emotional wellbeing and care.

4. Visitors who have been in contact with the patient before and during hospitalization are a possible source of MERS-CoV for other patients, visitors, and staff.

5. Visitors to MERS-CoV patients should be scheduled and controlled to allow for:

- Screening visitors for symptoms of acute respiratory illness before entering the hospital.

- Facilities should evaluate risk to the health of the visitor (e.g., visitor might have underlying illness putting them at higher risk for MERS-CoV) and ability to comply with precautions.

- Facilities should provide instruction, before visitors enter patients' rooms, on hand hygiene, limiting surfaces touched, and use of PPE according to current facility policy while in the patient's room.

- Facilities should maintain a record (e.g., log book) of all visitors who enter patient rooms.

- Visitors should not be present during aerosol-generating procedures.

- Visitors should be instructed to limit their movement within the facility.

- Exposed visitors (e.g., contact with symptomatic MERS-CoV patient prior to admission) should be advised to report any signs and symptoms of acute illness to their health care provider for a period of at least 14 days after the last known exposure to the sick patient.

\section{Implement engineering controls}

Consider designing and installing engineering controls to reduce or eliminate exposures by shielding HCP and other patients from infected individuals. Examples of engineering controls include physical barriers or partitions to guide patients through triage areas, curtains between patients in shared areas, closed suctioning systems for airway suctioning for intubated patients, as well as appropriate air-handling systems (with appropriate directionality, filtration, exchange rate, etc.) that are installed and properly maintained.

\section{Monitor and manage ill and exposed healthcare personnel}

1. HCP who care for patients with MERS-CoV should be monitored. They should immediately report any signs (e.g., fever ${ }^{1}$ ) or symptoms (e.g., cough, shortness of breath) of acute illness to their supervisor or a facility designated person (e.g., occupational health services) for a period of 14 days after the last known contact with a MERS CoV patient, regardless of their use of PPE.

2. HCP who develop any respiratory symptoms after an unprotected exposure (i.e., not wearing recommended PPE at the time of contact) to a patient with MERS-CoV should not report for work or should immediately stop working. These HCP should notify their supervisor, implement respiratory hygiene and cough etiquette, seek prompt medical evaluation, and comply with work exclusion until they are no longer deemed infectious to others.

3. For asymptomatic HCP who have had an unprotected exposure (i.e., not wearing recommended PPE at the time of contact) to a patient with MERS-CoV, exclude from work for 14 days to monitor for signs and symptoms of respiratory illness and fever. 
4. If necessary to ensure adequate staffing of the facility, the asymptomatic provider could be considered for continuing patient care duties after discussion with local, state, and federal public health authorities.

5. Facilities and organizations providing healthcare should:

- Implement sick leave policies for HCP, including contract staff and part-time personnel, that are non-punitive, flexible and consistent with public health guidance (e.g., policies should ensure ill HCP who may have MERS-CoV infection stay home, unless hospital admission for isolation and treatment is recommended).

- Ensure that all HCP are aware of the sick leave policies.

6. Provide employee health services that:

- Ensure that HCP have ready access, including via telephone, to medical consultation and, if needed, prompt treatment.

\section{Train and educate healthcare personnel}

1. Provide all HCP with job- or task-specific education and training on preventing transmission of infectious agents, including refresher training.

2. HCP must be medically cleared, trained, and fit tested for respiratory protection device use (e.g., N95 filtering face piece respirators), or medically cleared and trained in the use of an alternative respiratory protection device (e.g., Powered Air-Purifying Respirator, PAPR) whenever respirators are required.

3. Ensure that HCP are educated, trained, and have practiced the appropriate use of PPE prior to caring for a patient, including attention to correct use of PPE and prevention of contamination of clothing, skin, and environment during the process of removing such equipment.

\section{Implement environmental infection control}

1. Ensure that cleaning and disinfection procedures are followed consistently and correctly.

2. Standard cleaning and disinfection procedures (e.g., using cleaners and water to pre-clean surfaces prior to applying an EPA-registered disinfectant to frequently touched surfaces or objects for appropriate contact times as indicated on the product's label) are appropriate for MERS-CoV in healthcare settings, including those patient-care areas in which aerosol-generating procedures are performed. If there are no available EPA-registered products that have a label claim for MERS-CoV, products with label claims against human coronaviruses should be used according to label instructions. Management of laundry, food service utensils, and medical waste should also be performed in accordance with routine procedures.

\section{Establish reporting within hospitals and to public health authorities}

1. Implement mechanisms and policies that promptly alert key facility staff including infection control, healthcare epidemiology, hospital leadership, occupational health, clinical laboratory, and frontline staff about suspected or known MERS-CoV patients.

2. Communicate and collaborate with public health authorities.

- $\quad$ Promptly notify public health authorities of suspected or known patients with MERS-CoV.

- Facilities should designate specific persons within the healthcare facility who are responsible for communication with public health officials and dissemination of information to HCP. 


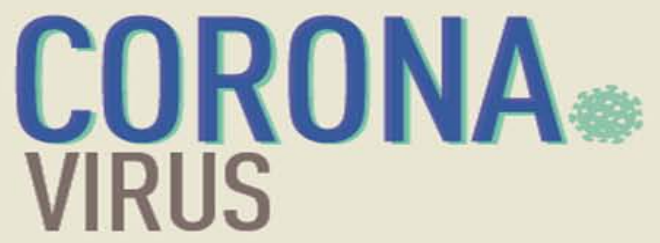

Middle East Respiratory Syndrome Coronavirus (MERS-CoV)

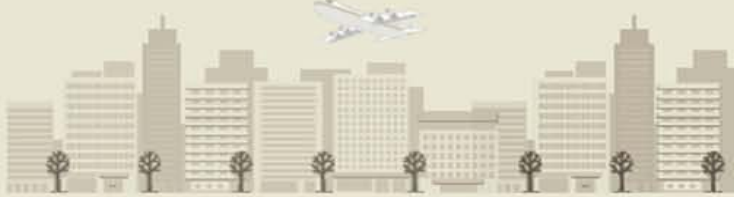

According to the recommendations of the specialists and scientists who attended the International Medical Meeting in Riyadh

Fighting Infections (of Corona and other acute respiratory infections) in Workplaces

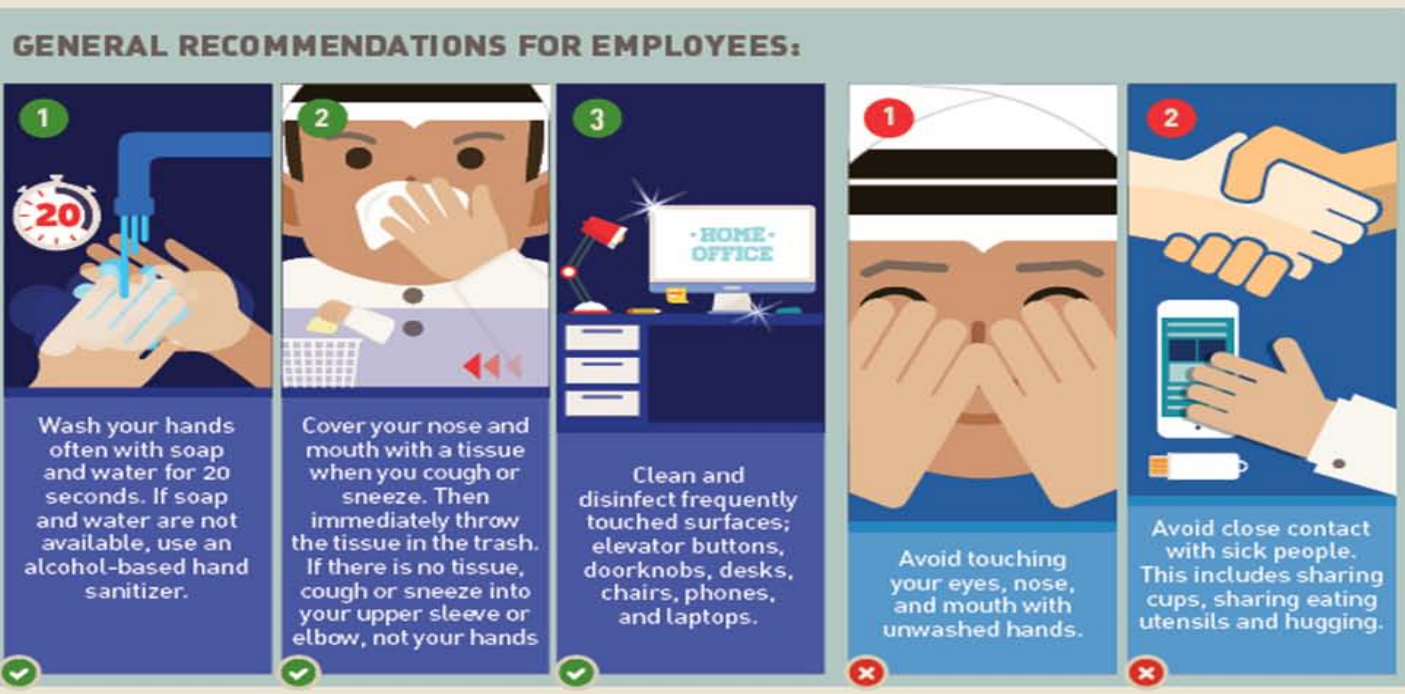

WHAT TO DO IF YOU FEEL SICK (FEVER, COUGH, SORE THROAT AND DIFFICULTY BREATHING]

If fever and respiratory symptoms (difficulty breathingl occur stay at home. Do not go back to work until free of symptoms for at least 24hrs
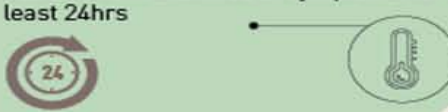

(24)

If you are diagnosed with
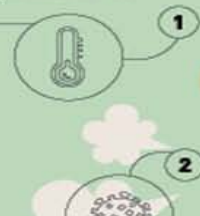

MERS CoV notify HR Do not come to work until 48 hours after you have recovered. You must test negative and be symptom free before returning to the workplace.

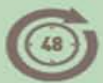

(1)

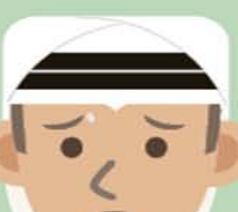

(2) Ensure all shared spaces are

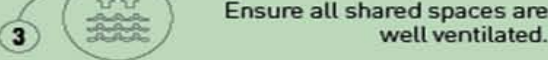

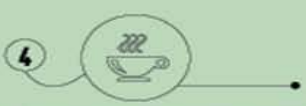

Do not share eating utensils, cups, towels, or washcloths.

5

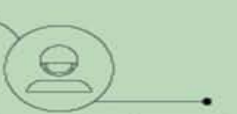

Employees should avoid contact with patients and their personal items, and use face-masks only if they are sick rvisiting sick patients

Do you have any questions ?

(8) $/ \mathrm{MOHPorta}$

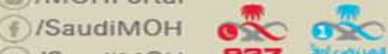

(9) ISaudiMOH $\mathbf{9 3 7}$ youm

www moh gov.sa/coronanew

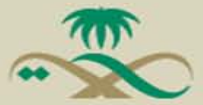

وزارة الصحة 


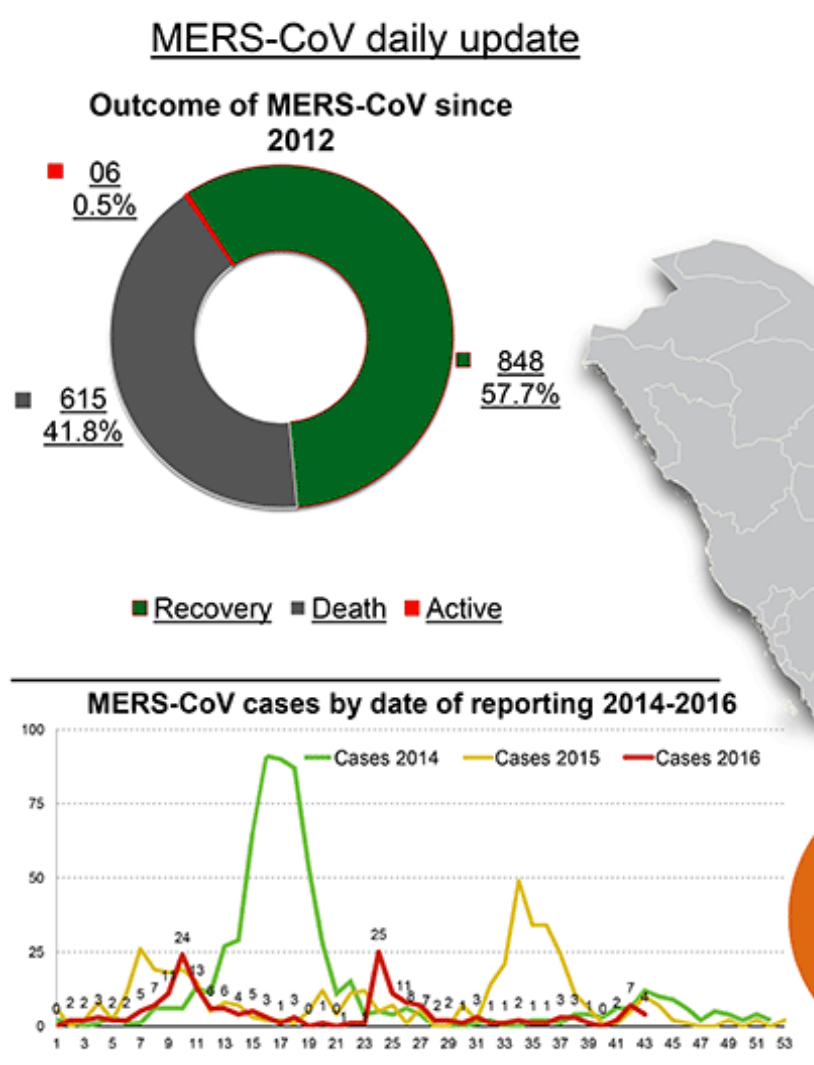

\begin{tabular}{|c|c|c|c|c|c|}
\hline & & (8) & $\mathrm{Ar}$ & $?$ & Total \\
\hline 0 & New symptomatic: & 0 & 0 & 0 & 0 \\
\hline 0 & New Asymptomatic: & 0 & 0 & 0 & 0 \\
\hline 0 & \multicolumn{4}{|l|}{ Deaths in last $24 \mathrm{~h}:$} & 0 \\
\hline 0 & \multicolumn{4}{|l|}{ Recovery in last $24 \mathrm{~h}$ : } & 0 \\
\hline
\end{tabular}

\section{Statistics}

7 Cases Under Treatment

847 Cases Recovered

The total number of confirmed MERSCOV cases is 1437+ 33 asymptomatic cases

As of 13 pm October 28,2016

* Since 2012. 615 cases passed away (May Allah have mercy upon them).

Disclaimer: The total number of cases is subject to change due to reclassification, retrospective investigation, consolidation of cases and laboratory data, and enhanced surveillance outcome.

\section{Comparisons}

Comparison of Saudi Arabia and South Korean outbreak of the Middle East respiratory syndrome coronavirus (mers-cov) infection.

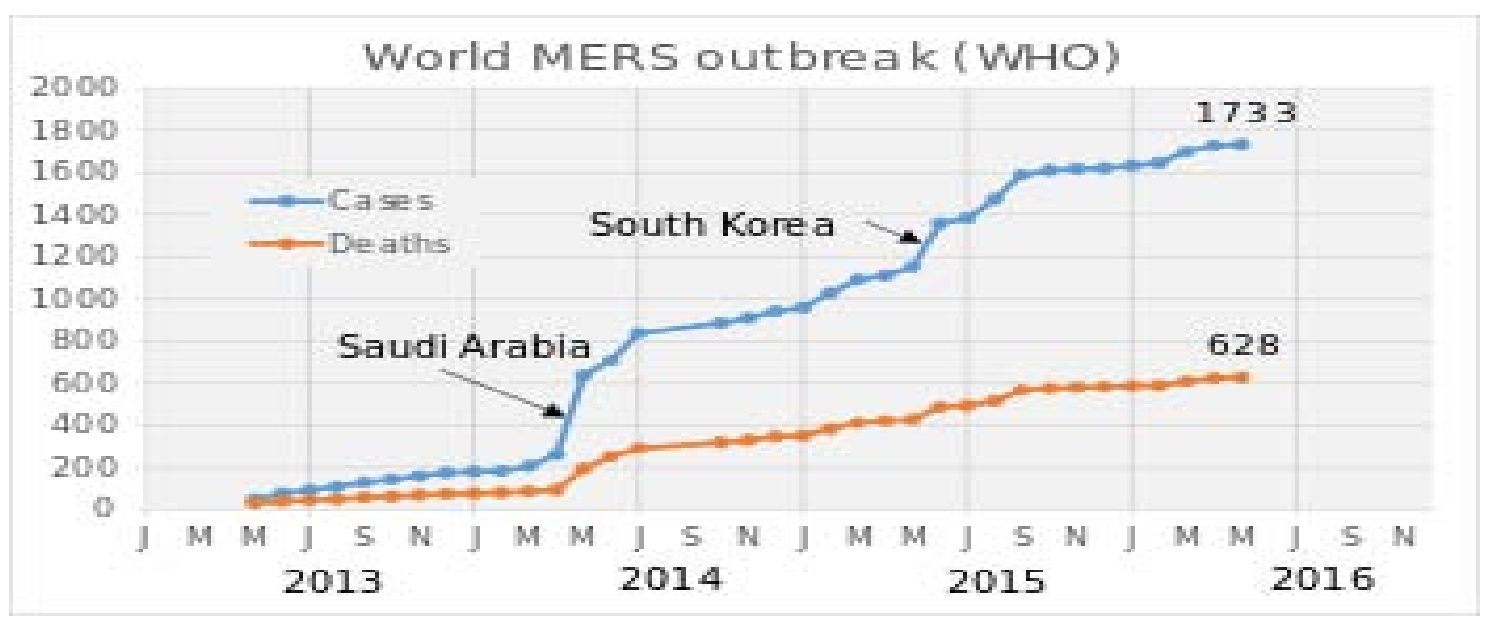


Texila International Journal of Public Health

Volume 4, Issue 4, Dec 2016

\begin{tabular}{|c|c|c|}
\hline & Saudi Arabia outbreak & South Korea outbreak \\
\hline Geographical location & Middle East Asia & Far East Asia \\
\hline City/Province & Riyadh, Jeddah & $\begin{array}{l}\text { Seoul, Daejeon, Gyeonggi } \\
\text { province }\end{array}$ \\
\hline Period & 11 Apr - 9 June, 2014 & $\begin{array}{l}\text { 4 May - Present (June 15), } \\
2015\end{array}$ \\
\hline Overall case number & 402 & 150 \\
\hline Health-care personnel (\%) & $27 \%$ & $17 \%$ \\
\hline Main transmission routes & $\begin{array}{l}\text { Health-care center } \\
\text { associated }\end{array}$ & Health-care center associated \\
\hline $\begin{array}{l}\text { Previous MERS case before } \\
\text { outbreak }\end{array}$ & Yes & No \\
\hline $\begin{array}{l}\text { Types of secondary exposure } \\
\text { who were not health-care } \\
\text { personnel }\end{array}$ & & \\
\hline $\begin{array}{l}\text { Admission to health-care } \\
\text { facility }\end{array}$ & $34 \%$ & $30 \%$ \\
\hline Emergency department & $8 \%$ & $49 \%$ \\
\hline $\begin{array}{l}\text { Visit to patient at health-care } \\
\text { facility }\end{array}$ & $17 \%$ & $29 \%$ \\
\hline $\begin{array}{l}\text { Annual outpatient } \\
\text { department visit (per } \\
\text { individual) }\end{array}$ & 4.5 & 14.6 \\
\hline $\begin{array}{l}\text { Annual number of hospital } \\
\text { admission (per } 100 \\
\text { individuals) }\end{array}$ & 10.5 & 16.1 \\
\hline
\end{tabular}

\section{Summary and recommendations}

A novel coronavirus, Middle East respiratory syndrome coronavirus (MERS-CoV) causing severe respiratory illness emerged in 2012 in Saudi Arabia. Many additional cases and clusters of MERS$\mathrm{CoV}$ infections have been detected subsequently in the Arabian Peninsula, particularly in Saudi Arabia Cases have also been reported from other regions, including North Africa, Europe, Asia, and North America. In countries outside of the Arabian Peninsula, patients developed illness after returning from the Arabian Peninsula or through close contact with infected individuals.

The number of cases in the Arabian Peninsula increased dramatically in March and April 2014 then declined sharply in ensuing months. However, cases continue to be detected. A large outbreak occurred in South Korea from May until early July 2015; the index case was an individual who had traveled to the Arabian Peninsula. Another large outbreak began in a hospital in Riyadh, Saudi Arabia, in the summer of 2015. As of mid-September 2015, it continues.

MERS-CoV is closely related to coronaviruses found in bats, suggesting that bats might be a reservoir of MERS-CoV. Camels likely serve as hosts for MERS-CoV. The presence of case clusters strongly suggests that human-to-human transmission occurs. 
Real-time reverse-transcriptase polymerase chain reaction (rRT-PCR) testing applied to respiratory secretions is the diagnostic assay of choice. Ideally, lower respiratory tract, upper respiratory tract, and serum samples should be obtained. Lower respiratory tract specimens should be a priority for collection and testing. To increase the likelihood of detecting MERS-CoV, we suggest collection of multiple specimens from different sites and at different times.

Individuals with an acute respiratory infection who have an epidemiologic link to MERS-CoV or who have had an unusual or unexpected clinical course (especially sudden deterioration despite appropriate treatment) should be tested for MERS-CoV. Certain other patients may also require evaluation for MERS-CoV infection. Specific recommendations regarding whom to test are presented above.

There is currently no treatment recommended for coronavirus infections except for supportive care as needed.

An increased level of infection control precautions is recommended when caring for patients with probable or confirmed MERS-CoV infection compared with that used for patients with communityacquired coronaviruses or other community-acquired respiratory viruses. The United States Centers for Disease Control and Prevention (CDC) recommends the use of standard, contact, and airborne precautions for the management of hospitalized patients with known or suspected MERS-CoV infection. (See 'Infection control' above.)

There is no licensed vaccine for MERS-CoV.

The World Health Organization (WHO) does not recommend either special screening for MERS$\mathrm{CoV}$ at points of entry or the application of any travel or trade restrictions. The Ministry of Health of Saudi Arabia recommended that, in 2014, older adults, those with chronic diseases, immunocompromised patients, pregnant women, and children postpone their plans to travel to Mecca, Saudi Arabia, for Hajj and/or Umrah.

\section{References}

[1]. https://en.wikipedia.org/wiki/Middle_East_respiratory_syndrome_coronavirus

[2]. http://www.moh.gov.sa/en/CCC/PressReleases/Pages/default.aspx

[3]. http://www.moh.gov.sa/en/CCC/informationcenter/pages/healthguidelines4.aspx

[4]. http://www.cdc.gov/coronavirus/mers/about/transmission.html

[5]. http://www.cdc.gov/coronavirus/mers/infection-prevention-control.html

[6]. http://www.thelancet.com/action/showFullTextImages?pii=S0140-6736\%2815\%2960454-8

[7]. http://www.cdc.gov/coronavirus/mers/faq.html

[8]. http://www.cdc.gov/coronavirus/mers/about/symptoms.html

[9]. http://www.uptodate.com/contents/image?imageKey=ID/90331\&topicKey=ID/89705\&source=outline_lin $\mathrm{k} \&$ search=\&utdPopup=true

[10]. odate.com/contents/image?imageKey=ID/90331\&topicKey=ID/89705\&source=outline_link\&search=\&ut dPopup=true

[11]. https://en.wikipedia.org/wiki/Middle_East_respiratory_syndrome\#Treatment 